\title{
The Use of Tooth Derived Bone Graft Materials in Sinus Augmentation Procedures: a Systematic Review
}

\author{
Eliyahu Shavit ${ }^{1}$, Ilana Shavit ${ }^{1}$, Daniel Pinchasov ${ }^{1}$, David Shavit ${ }^{1}$, Ginnady Pinchasov ${ }^{1}$, Gintaras \\ Juodzbalys ${ }^{1}$
}

${ }^{1}$ Department of Oral and Maxillofacial Surgery, Lithuanian University of Health Sciences, Lithuania.

\author{
Corresponding Author: \\ Eliyahu Shavit \\ Heh Be'iyar 11, Ashdod \\ Israel \\ Phone: +37062579538 \\ E-mail: eli.shavit4@gmail.com
}

\begin{abstract}
Objectives: The goal of this systematic review was to assess the current literature about sinus augmentation procedure using different types of tooth derived bone graft materials, thorough analysing the outcomes of sinus grafting with tooth grafts compared to sinus grafting with xenografts, allografts and alloplasts by radiography and histomorphometry.

Material and Methods: An electronic search in the MEDLINE (NCBI PubMed and PubMed Central) database was conducted to identify articles concerning application of tooth bone grafts in sinus augmentation. The search was restricted to English language articles published in the last 10 years (December 2009 to March 2019).

Results: In total, 21 articles were found, out of which 7 met the inclusion criteria and were included in the final synthesis. According to the type of diagnostic tool, data about graft material outcome in sinus was extracted, and included the residual alveolar height, augmented graft height, resorption height as seen in panoramic radiography and histomorphometric analysis of new bone formation and residual graft material.

Conclusions: Within the limitations of our review, we suggest that tooth derived graft materials are as successful as xenografts, allografts and alloplasts in sinus augmentation procedures according to the radiographic and histomorphometric showings. Additional wider research should be conducted in order to determine whether tooth derived graft materials are superior to the currently used materials.
\end{abstract}

Keywords: bone formation; bone transplantation; maxillary sinus; radiography; tooth.

\author{
Accepted for publication: 26 June 2019 \\ To cite this article: \\ Shavit E, Shavit I, Pinchasov D, Shavit D, Pinchasov G, Juodzbalys G. \\ The Use of Tooth Derived Bone Graft Materials in Sinus Augmentation Procedures: a Systematic Review \\ J Oral Maxillofac Res 2019;10(2):e1 \\ URL: http://www.ejomr.org/JOMR/archives/2019/2/e1/v10n2e1.pdf \\ doi: $\underline{10.5037 / \text { jomr.2019.10201 }}$
}




\section{INTRODUCTION}

Three million people in the United States only, have implants, and 500,000 more are joining the statistics each year, according to the American Academy of Implant Dentistry [1]. When bone loss occurs after tooth extraction in the posterior maxillary area and implant restoration is planned, a unique problem arises due to the presence of the maxillary sinus [2]. Implant placement is impossible when maxilla is severely resorbed in this region [3]. The most common procedure to overcome this problem is sinus elevation with bone augmentation, thus increasing the vertical bone height [4].

Various materials have been used over the years as bone grafts; those include allografts, xenografts, alloplasts and autogenous bone. Ideally, bone graft material should have 3 qualities:

1. The ability to promote formation of bone tissue by inducing differentiation of progenitor cells into osteoblasts - osteoinduction [5].

2. Providing the framework, on which osteoblasts spread and form the new bone - osteoconduction []․

3. Stimulation of bone generation by inducing the cells which are present in the graft material osteoproliferation [6].

The material which is currently considered ideal is autogenous bone, since it contains all 3 properties for the optimal bone graft material mentioned above. Nonetheless, it has shortcomings such as donor site infection risk, limited available amount, and notable resorption rates [6]. Allografts, xenografts and alloplasts are not amount restricted, do not create donor site morbidity and have the capacity to carry cellular growth factors [4]. Yet still, none of them exhibits all the 3 properties, since xenografts and alloplasts have only osteoconductive capacity and allografts fail to promote osteoproliferation [6].

Lately, the use of permanent teeth as component for bone graft materials was introduced, and it was confirmed histologically and clinically by some studies [6-8]. Autogenous tooth bone graft material (autoBT) was developed in 2008 and has been used for guided bone regeneration to support implant placement. The condition of extracted teeth determines the amount of bone graft obtained, and its histological properties were shown to be similar to those of autogenous bone grafts. The maxillary sinus is frequently selected for examining the healing process of different bone graft materials, since it is not a naturally bone forming area, and because the sinus cavity is a contained-type defect [9].
The chemical composition of teeth is very similar to this of bones. Dentin shows the most similar composition with $65 \%$ inorganic substances and 35\% organic substances - the same composition bones have. Also, teeth and maxillary bones originate from one embryologic source - the neural crest. The organic substances within dentin include collagen type I as well as bone morphogenetic proteins (BMPs) and non-collagenous proteins (NCPs) - which are known to induce bone resorption and generation [10].

The aim of the present article is to systematically review the success and reliability of utilizing teeth as a source for bone graft material in sinus augmentation procedure, by observing the outcomes as seen in radiological, histological and histomorphometric analyses, compared to the widely used nowadays graft materials in sinus augmentation. The following outcomes are intended to be reviewed in the present paper: augmented graft height, graft height resorption, as well as the following histomorphometric results: new bone formation, residual graft material, bone marrow space, connective tissue area.

\section{MATERIAL AND METHODS Protocol}

This systematic review was conducted following the Preferred Reporting Items for Systematic Reviews and Meta-Analyses (PRISMA) statement for systematic reviews [11].

\section{Focus question}

The question was developed according to the Patient, Intervention, Comparison and Outcome (PICO) framework as described in Table 1.

\section{Types of publications}

The review included prospective and retrospective clinical studies in vivo published in English language.

\section{Types of studies}

The review included any clinical study in vivo that met the relevant criteria.

\section{Population}

The studies included human patients with atrophied posterior maxilla undergoing sinus elevation with bone graft materials produced from teeth, 
Table 1. Focus question according to the PICO framework

\begin{tabular}{l|l}
\hline Patient/problem & Patients with posterior maxillary region resorption who are planned to receive implant prosthesis, or tested animals. \\
\hline Intervention & Sinus augmentation using tooth material in different forms; powder, block. \\
\hline Comparison & Other bone graft materials used in sinus augmentation - allograft, xenograft, and alloplast. \\
\hline Outcome & $\begin{array}{l}\text { Gained bone height after grafting, stability of sinus graft height, bone formation and regeneration potential as shown } \\
\text { in histomorphometric analysis, implant stability, complications, implant survival. }\end{array}$ \\
\hline Focus question & $\begin{array}{l}\text { Is utilizing teeth as bone graft in sinus augmentation procedures can be considered as effective as currently used } \\
\text { bone grafts? }\end{array}$ \\
\hline
\end{tabular}

as well as experimented animals receiving sinus augmentation with tooth graft materials.

\section{Literature search strategy}

According to PRISMA guidelines [11] electronic search was conducted using MEDLINE (NCBI PubMed and PubMed Central [PMC]) online library in order to locate articles concerning the use of autogenous teeth as bone graft material in maxillary sinus augmentation procedure. The following keywords were searched: "autogenous tooth" AND "maxillary sinus". Demineralized tooth dentin AND maxillary sinus. Demineralized dentin matrix AND sinus augmentation. The search was restricted to English language articles published during the last 10 years, from December 2009 to March 2019.

\section{Inclusion and exclusion criteria Inclusion criteria for the selection}

The following inclusion criteria were assessed for selection of articles:

- Studies including at least 5 subjects (animal or human) undergoing sinus augmentation.

- Studies evaluating the use of tooth derived graft materials in the sinus augmentation procedure.

- Studies evaluating the tooth derived graft material by at least one of the following diagnostic tools: panoramic radiography, computed tomography, histological or histomorphometric analysis.

- Studies which assessed the tooth derived graft material by comparing to control groups receiving sinus grafting with other materials such as xenograft, allograft, and alloplast.

- Follow-up period of at least 4 months after grafting for human subjects and 8 weeks for animals.

\section{Exclusion criteria for the selection}

- Studies in which autogenous tooth was mixed with autogenous bone to graft the maxillary sinus.
- Studies which evaluated human patients without mentioning their medical condition.

- Technical notes.

- Case reports.

\section{Sequential search strategy}

The selected articles were subjected independently to clear inclusion and exclusion criteria by 2 reviewers as follows. After conducting initial MEDLINE (NCBI PubMed and PMC) literature search, articles with relevant titles were chosen, considering the exclusion criteria. Subsequently, exclusion of studies with irrelevant abstract data was done. In the last stage of screening, inclusion criteria were considered to confirm the eligibility of each study was after reading its full text.

\section{Date extraction}

Data in form of variables was independently extracted from studies, considering the aims and objectives of the present review, as indexed below.

\section{Data items}

- "Author" - indicates the corresponding authors.

- "Year" - the year of publication.

- "Study design" - describes the type of the study.

- "Number of subjects, model" - indicated the number of the investigated subjects, and whether human or animals were tested.

- "Number of implants" (of study and control groups) - indicating the number of dental implants that were placed, if any were.

- "Harvest material" (of study and control groups) describes the bone graft material which was used in study and in comparison groups.

- "Follow-up period" - indicates the outcomes follow-up period in months for human studies and weeks for animal studies.

- "Evaluation methods" - describes the tool which was used to investigate the outcome of sinus augmentation 
- "Outcomes" - relates to the radiographic, computed tomography, histomorphometric and histological results after maxillary sinus augmentation.

\section{Risk of bias}

In order to assess the quality of the included randomized clinical trials and identify flaws in the studies which can make the interventions' effect unclear or underestimated, Cochrane Collaboration's Tool for assessing risk of bias [12] was used. The results are summarized in Table 2.

\section{Statistical analysis}

Studies were not homogenous; therefore a metaanalysis could not be conducted. Data extracted from studies in forms of parameters were expressed as mean and standard deviation (M [SD]). Statistical significance level is described further in results section with correspondence to each reviewed study.

\section{RESULTS}

\section{Study selection}

The electronic database MEDLINE (NCBI PubMed and PMC) search yielded 21 results. After screening process which included reviewing the abstracts and titles of all identified results, 12 results were excluded due to irrelevant titles or abstract. Subsequently, assessment of 9 full text articles for eligibility considering the determined inclusion and exclusion criteria was performed, two studies were excluded - Jeong et al. [13] and Pohl et al. [14], as they were not meeting our criteria. Finally, seven studies were included in the review. The study selection process is presented in Figure 1.

\section{Risk of bias in individual studies}

Results of quality assessment of included studies are presented in Table 2.

Jun et al. [15] was considered to have low risk of bias, Jeong et al. [16] had unclear risk of bias, Kim et al. [17] had low risk of bias, and 4 other studies [18-21] had unclear risk of bias.

\section{Study characteristics}

The present review included 167 subjects (136 human, 26 rabbits and 5 mini-pigs) tested in 4 human studies and 3 animal model studies, which were published between 2013 and 2018. The study designs were as follows: 2 prospective observational studies (Jun et al. [15], Kim et al. [18]); 2 retrospective observational studies (Jeong et al. [16], Kim et al. [17]) and 3 animal clinical studies (Lee et al. [19], Sohn et al. [20], $\mathrm{Xu}$ et al. [21]). The follow-up period ranged from 4 months to 2 years in human studies and 8 to 12 weeks in animal studies. The characteristics are summarized in Table 3.

\section{Tooth derived graft material preparation}

Information about type of tooth derived bone graft materials used in each study is presented in Table 4.

\section{Radiographic findings Human studies}

Three studies [16-18] used radiography and one study [15] used computed tomography as evaluation tool.

The following measurements were the main:

- Preoperative residual alveolar height.

- Postoperative increase in bone height/augmented graft height.

- Bone resorption height after follow-up period.

Table 2. Risk of bias assessment

\begin{tabular}{|c|c|c|c|c|c|c|c|}
\hline Study & Year & $\begin{array}{c}\text { Random } \\
\text { sequence } \\
\text { generation }\end{array}$ & $\begin{array}{c}\text { Allocation } \\
\text { concealment }\end{array}$ & $\begin{array}{c}\text { Blinding of } \\
\text { participants and } \\
\text { personnel }\end{array}$ & $\begin{array}{l}\text { Blinding of } \\
\text { outcome } \\
\text { assessment }\end{array}$ & $\begin{array}{c}\text { Incomplete } \\
\text { outcome } \\
\text { data }\end{array}$ & $\begin{array}{l}\text { Selective } \\
\text { reporting }\end{array}$ \\
\hline Jun et al. [15] & 2014 & + & + & + & + & $?$ & $?$ \\
\hline Jeong et al. [16] & 2014 & - & $?$ & + & + & $?$ & $?$ \\
\hline Kim et al. [17] & 2014 & - & - & + & + & + & + \\
\hline Kim et al. [18] & 2016 & + & + & $?$ & $?$ & $?$ & - \\
\hline Lee et al. [19] & 2013 & - & - & + & + & + & - \\
\hline Sohn et al. [20] & 2018 & - & - & $?$ & $?$ & $?$ & $?$ \\
\hline $\mathrm{Xu}$ et al. [21] & 2018 & - & + & + & $?$ & $?$ & $?$ \\
\hline
\end{tabular}




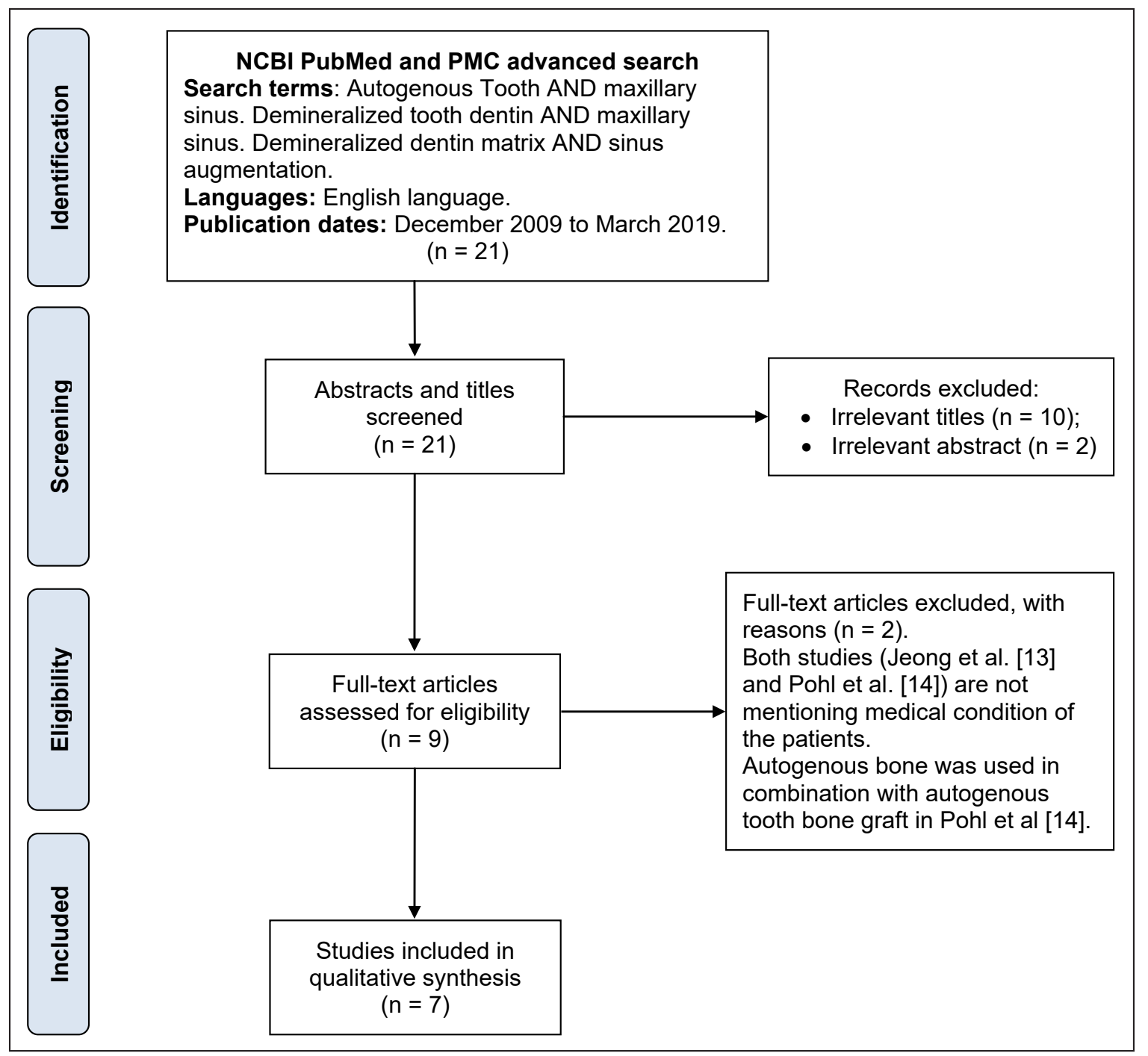

Figure 1. PRISMA flow diagram.

In 2014, Jun et al. [15] studied 43 participants with reduced residual bone height (less than $5 \mathrm{~mm}$ ), which were divided to control group and experimental group and underwent sinus augmentation with anorganic bovine bone (Bio-Oss ${ }^{\circledR}$; Geistlich Pharma AG, Wolhusen, Switzerland) and autoBT powder, respectively. Initial preoperative features of all participants were recorded by preoperative computed tomography, and 4 months postoperatively computed tomography was made. No significant difference between the groups in preoperative residual bone height was detected, as well as in augmented graft height. Furthermore, this study measured the pre and postoperative sinus membrane thickness, a measurement which yielded no significant difference between the groups.

In a study conducted by Jeong et al. in 2014 [16], the researchers examined 30 maxillary sinuses of 26 patients, which were divided to 3 groups and received autoBT, demineralized freeze-dried bone allograft (DFDBA) or deproteinized bovine bone mineral (DBBM) sinus grafting. The information was collected prior to surgery in order to measure the residual bone height, than to measure the height of grafted material immediately after surgery, and six months after surgery. After 6 months, there was no significant difference in the average resorption ratio; $13.57 \%$ for autoBT group, $14.3 \%$ for DFDBA group and $11.92 \%$ for DBBM group $(\mathrm{P}=0.649)$ as well as in average resorption height $(\mathrm{P}=0.576)$ between the groups.

Kim et al. [17] checked in 2014 the amount of bone resorption around implants by comparing autoBT and synthetic bone graft after crestal approach sinus lift and simultaneous implantation. 37 participating patients were divided into 2 groups, in 17 patients autoBT was used for sinus augmentation and in 20 remaining synthetic bone (Osteon ${ }^{\mathrm{TM}}$ II, Genoss Co., Suwon, Korea) grafting for sinus elevation was done. A panoramic X-ray was prior to surgery, immediately after surgery and 1 year post operatively. 
Table 3. Study characteristics

\begin{tabular}{|c|c|c|c|c|c|c|c|c|c|c|c|}
\hline \multirow[b]{2}{*}{ Study } & \multirow[b]{2}{*}{ Study design } & \multicolumn{2}{|c|}{ No. of subjects } & \multirow[b]{2}{*}{ Model } & \multicolumn{2}{|c|}{ No. of implants } & \multicolumn{2}{|c|}{ Harvest material } & \multirow[b]{2}{*}{$\begin{array}{c}\text { Follow-up } \\
\text { period }\end{array}$} & \multirow[b]{2}{*}{ PR/CT } & \multirow[b]{2}{*}{$\begin{array}{l}\text { Histological } \\
\text { HMM }\end{array}$} \\
\hline & & Total & $\begin{array}{c}\text { Tooth graft } \\
\text { group }\end{array}$ & & Total & $\begin{array}{c}\text { Tooth graft } \\
\text { group }\end{array}$ & Study group & Comparison group & & & \\
\hline Jun et al. [15] & Prospective study & 43 & 22 & Human & 57 & 29 & AutoBT & Bio-Oss ${ }^{\circledR}$ & 4 months & + & + \\
\hline Jeong et al. [16] & Retrospective comparative & 26 & 8 & Human & \multicolumn{2}{|r|}{$\mathrm{N} / \mathrm{A}$} & AutoBT & DFDBA, DBBM & 6 months & + & - \\
\hline Kim et al. [17] & Retrospective comparative & 37 & 17 & Human & 34 & 18 & AutoBT & Synthetic bone grafts & 12 months & + & - \\
\hline Kim et al. [18] & Prospective & 30 & 15 & Human & 59 & 28 & AutoFDT block + PRP & Allograft and xenograft powder + PRP & 24 months & + & + \\
\hline Lee et al. [19] & Animal clinical & \multicolumn{2}{|r|}{5} & Minipigs & \multicolumn{2}{|r|}{$\mathrm{N} / \mathrm{A}$} & AutoBT & Synthetic hydroxyapetite & 12 weeks & - & + \\
\hline Sohn et al. [20] & Animal clinical & \multicolumn{2}{|r|}{18} & Rabbits & \multicolumn{2}{|r|}{ N/A } & DTD & Blood clots alone, $\beta-\mathrm{TCP}$ & $2,4,8$ weeks & - & + \\
\hline Xu et al. [21] & Animal experimental & \multicolumn{2}{|r|}{8} & Rabbits & & $\mathrm{N} / \mathrm{A}$ & Demineralized particulate human tooth & Bio-Oss $^{\circledR}$ & 2,8 weeks & - & + \\
\hline
\end{tabular}

HMM = histomorphometric; $\mathrm{PR}=$ panoramic radiography; $\mathrm{CT}=$ computed tomography; $\mathrm{PRP}=$ platelet rich plasma; autoBT $=$ autogenous tooth bone graft; autoFDT $=$ autogenous fresh demineralized tooth; DTD $=$ demineralized tooth dentin DFDBA = demineralized freeze-dried bone allograft; DBBM = deproteinized bovine bone mineral; $\beta-\mathrm{TCP}=\beta$-tricalcium phosphate; - - not indicated.

Table 4. Tooth derived graft materials characteristics

\begin{tabular}{|c|c|c|c|c|}
\hline Study & Source & Pre-fabrication procedures & Demineralization & Form \\
\hline Jun et al. [15] & Autogenous teeth & $\begin{array}{l}\text { Teeth kept refrigerated or frozen, soft tissues removal, } \\
\text { tooth sectioning to crown and root, pulp tissue removal }\end{array}$ & - & $\begin{array}{l}\text { AutoBT powder } \\
(0.5-1 \mathrm{~mm})\end{array}$ \\
\hline Jeong et al. [16] & Autogenous teeth & - & - & AutoBT powder \\
\hline Kim et al. [17] & Autogenous teeth & - & - & AutoBT powder \\
\hline Kim et al. [18] & Autogenous teeth & $\begin{array}{l}\text { Remnant soft tissues removal, pulp tissues removal, } \\
\text { making many small holes on tooth surface }\end{array}$ & $\begin{array}{l}\text { Was performed in an ultrasonic chamber } \\
\text { equipped with vacuum and cooling units. }\end{array}$ & AutoFDT block \\
\hline Lee et al. [19] & Autogenous teeth & $\begin{array}{l}\text { Removal of all extraneous material, tooth sectioning } \\
\text { to crown and root, pulp tissue removal }\end{array}$ & $30 \mathrm{~min}$ immersion in $0.6 \mathrm{~N}$ hydrochloric acid solution at $2{ }^{\circ} \mathrm{C}$ & $\begin{array}{l}\text { Autogenous tooth powder } \\
(400-800 \mu \mathrm{m})\end{array}$ \\
\hline Sohn et al. [20] & Extracted permanent teeth ${ }^{\mathrm{a}}$ & Attached soft tissues were removed & $\begin{array}{l}15 \text { min in } 0.6 \mathrm{~N} \text { hydrochloride under vacuum compression } \\
\text { and ultrasonic vibration. }\end{array}$ & $\begin{array}{l}\text { DTD powder } \\
(0.8-1 \mathrm{~mm})\end{array}$ \\
\hline Xu et al. [21] & Extracted human permanent teet ${ }^{b}$ & Attached soft tissues were removed & $\begin{array}{l}15 \mathrm{~min} \text { in } 0.6 \mathrm{~N} \text { hydrochloride under vacuum compression } \\
\text { and ultrasonic vibration. }\end{array}$ & $\begin{array}{l}\text { DHT powder } \\
(0.8-1 \mathrm{~mm})\end{array}$ \\
\hline
\end{tabular}

${ }^{a}$ Not indicated whether the teeth were autogenous.

Non autogenous teeth were used - human teeth in rabbit's maxilla

DHT = demineralized human tooth; DTD = demineralized tooth dentin; autoFDT = autogenous fresh demineralized tooth; autoBT = autogenous tooth bone graft; - = not indicated.

Table 5. Outcomes evaluation by panoramic radiography or computed tomography

\begin{tabular}{|c|c|c|c|c|c|c|c|c|c|c|c|c|}
\hline \multirow{2}{*}{ Study } & \multirow{2}{*}{ Materials used } & \multirow{2}{*}{$\begin{array}{l}\text { Type of sinus } \\
\text { lift }\end{array}$} & \multicolumn{2}{|c|}{ Residual alveolar height (mm) } & \multirow{2}{*}{$\begin{array}{l}\text { Statistical } \\
\text { difference }\end{array}$} & \multicolumn{2}{|c|}{ Average augmented graft height (mm) } & \multirow{2}{*}{$\begin{array}{l}\text { Statistical } \\
\text { difference }\end{array}$} & \multicolumn{2}{|c|}{ Average resorption height (mm) } & \multirow{2}{*}{$\begin{array}{c}\text { Follow-up } \\
\text { (months) }\end{array}$} & \multirow{2}{*}{$\begin{array}{l}\text { Statistical } \\
\text { difference }\end{array}$} \\
\hline & & & Study group & Comparison group & & Study group & Comparison group & & Study group & Comparison group & & \\
\hline Jun et al. [15] & $\begin{array}{c}\text { Study group: } \\
\text { autoBT; } \\
\text { Comparison group: } \\
\text { Bio-Oss }^{\circledR}\end{array}$ & $\begin{array}{l}\text { Lateral window } \\
\text { approach }\end{array}$ & 3.12 (SD 1.17) & 3.17 (SD 1.26) & $\begin{array}{l}\text { Not significant } \\
(\mathrm{P}=0.889)\end{array}$ & 10.73 (SD 2.08) & 10.45 (SD 2.56) & $\begin{array}{l}\text { Not significant } \\
(P=0.709)\end{array}$ & Not evaluated & Not evaluated & 4 & Not evaluated \\
\hline Jeong et al. [16] & $\begin{array}{c}\text { Study group: } \\
\text { autoBT; } \\
\text { Comparison group: } \\
\text { DFDBA, DBBM }\end{array}$ & $\begin{array}{l}\text { Lateral window } \\
\text { approach }\end{array}$ & $3.3-10$ & $\begin{array}{l}\text { DFDBA: } 1.7-10.6 ; \\
\text { DBBM: } 4.9-10.3\end{array}$ & Not evaluated & 9.07 (SD 2.92) & $\begin{array}{l}\text { DFDBA: } 10.95 \text { (SD 2.75); } \\
\text { DBBM: } 11.83 \text { (SD 2.81) }\end{array}$ & $\begin{array}{l}\text { Not significant } \\
(\mathrm{P}=0.182)\end{array}$ & 1.27 (SD 1.06) & $\begin{array}{l}\text { DFDBA: } 1.53 \text { (SD 0.71); } \\
\text { DBBM: } 1.37 \text { (SD 1.09) }\end{array}$ & 6 & $\begin{array}{l}\text { Not significant } \\
(\mathrm{P}=0.576)\end{array}$ \\
\hline Kim et al. [17] & $\begin{array}{c}\text { Study group: } \\
\text { autoBT; } \\
\text { Comparison group: } \\
\text { Osteon }{ }^{\text {nem }} \\
\end{array}$ & $\begin{array}{l}\text { Crestal } \\
\text { approach }\end{array}$ & 9.64 & 9.22 & $\begin{array}{l}\text { Not significant } \\
(\mathrm{P}=0.973)\end{array}$ & 4.89 & 6.22 & $\begin{array}{l}\text { Not significant } \\
\quad(P=0.46)\end{array}$ & 0.76 & 0.53 & 12 & $\begin{array}{l}\text { Not significant } \\
\quad(\mathrm{P}=0.57)\end{array}$ \\
\hline Kim et al. [18] & $\begin{array}{c}\text { Study group: } \\
\text { autoFDT + PRP; } \\
\text { Comparison group: } \\
\text { allograft + xenograft + PRP } \\
\end{array}$ & $\begin{array}{l}\text { Lateral window } \\
\text { approach }\end{array}$ & $1.2-4.2$ & $1.55-3.85$ & $\begin{array}{l}\text { Not significant } \\
(\mathrm{P}=0.233)\end{array}$ & $11.62(\mathrm{SD} 2.22)$ & 13.65 (SD 1.35) & $\begin{array}{l}\text { Significant } \\
(\mathrm{P}=0.007)\end{array}$ & $1.23(\mathrm{SD} 0.73)$ & $1.77(\mathrm{SD} 0.54)$ & 24 & $\begin{array}{l}\text { Not significant } \\
(\mathrm{P}=0.021)\end{array}$ \\
\hline
\end{tabular}

DFDBA = demineralized freeze-dried bone allograft; DBBM = deproteinized bovine bone mineral; autoFDT = autogenous fresh demineralized tooth; autoBT = autogenous tooth bone graft; PRP = platelet-rich plasma. 
There was no significant difference in residual alveolar height (distance from crest to sinus floor) presurgically $(\mathrm{P}=0.973)$, and in the mean increase of bone height $(\mathrm{P}=0.46)$ immediately postsurgery. No statistically significant difference was noticed in amount of bone graft material resorption between the

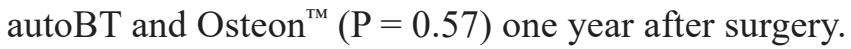
In 2016, Kim et al. [18] effectiveness of autogenous tooth in a form of fresh demineralized tooth (autoFDT) block with platelet rich plasma (PRP) for sinus maxillaris elevation was evaluated. Thirty patients with less than $5 \mathrm{~mm}$ residual alveolar height were sorted to 2 groups, 15 patients in Group I underwent sinus elevation with autoFDT block with PRP and 15 other patients in Group II underwent sinus elevation with combined graft (allograft and xenograft) powder and PRP. During a follow-up period of 2 years after installing the final prosthesis, clinical and radiological assessment of graft material amount, residual alveolar height, sinus height after grafting, augmented graft height and resorption height was done. There was no significant difference statistically between 2 groups in preoperative residual alveolar. The radiologic evaluation showed significant difference in augment graft height, however, resorption height of the grafts during the follow-up period was not significantly different between groups. The radiographic and CT results are summarized and values are indicated in Table 5.

\section{Histomorphometric findings}

Two human studies $[\underline{15}, \underline{18}]$ and 3 animal studies [19-21] used histomorphometric analysis as a tool to evaluate the bone formation capacity of tooth bone grafts. The histomorphometric analysis results are summarized in Table 6 and 7.

\section{Human studies}

In the study of Jun et al. [15], histmorphometry revealed no difference in new bone formation percentage between groups with $26.49(7.13) \%$ in Bio-Oss ${ }^{\circledR}$ group and 31.07 (14.52)\% in autoBT group, in residual graft material percentage with 31.12 $(14.51) \%$ in Bio-Oss ${ }^{\circledR}$ group and $29(10.27) \%$ in autoBT group, and in marrow space percentage with 42.38 (16.37)\% for Bio-Oss ${ }^{\circledR}$ and 39.93 (18.92)\% for autoBT. The parameter which showed statistically significant difference between groups was mean osteoid thickness which was $8.35 \mu \mathrm{m}$ in Bio-Oss ${ }^{\circledR}$ group and $13.12 \mu \mathrm{m}$ in autoBT group $(\mathrm{P}=0.025)$.

In another study, which was conducted by Kim et al. [18], new bone formation was $23.13(1.42) \%$ in Group 1 (autoFDT block + PRP) and 24.18 (2.19)\% in Group 2 (combined allograft and xenograft powder + PRP), no difference between groups. Significant difference was noticed in the area of residual graft material between groups, with 22.21 (1.19)\% (Group 1) and 31.18 (2.09)\% (Group 2). Larger spaces were observed between the blocks in comparison to those between powders.

\section{Animal studies}

In a study of Lee et al. in Korea in 2013 [19], the maxillary sinuses of 5 mini-pigs were experimented. The right maxillary sinus of those mini-pigs were augmented with graft material which was produced from own extracted teeth, while the left sinuses were grafted using synthetic hydroxyapatite. The difference in ratio of bone formation (new bone/total bone) between the experimental and control group was not statistically significant due to the small number of experimented subjects, however, better results were observed in experimental group showing 57.19 $(11.16) \%$ in the autogenous teeth group and 34.07 $(13.09) \%$ in the synthetic hydroxyapatite group.

Sohn et al. [20] compared bone formation capacity of demineralized tooth dentin (DTD) to other bone graft materials (blood clots alone, Bio-Oss ${ }^{\circledR}, \beta$-tricalcium phosphate). They filled maxillary sinuses of 18 adult male rabbits in 4 different groups with those graft materials and performed histomorphometric analysis at 2, 4, and 8 weeks after grafting. In DTD group, the new bone/area of augmented sinus ratio showed significantly greater values at 8 weeks $(28.09[1.51] \%)$ compared to 2 weeks (17.71 [2.2]\%) and 4 weeks $(20.73[1.99] \%)$. Also, this ratio as significantly higher in comparison with Group 3 ( $\beta$-tricalcium phosphate) after 8 weeks. Total bone area (mature lamellar bone + new bone) was also significantly higher in Group 4 (DTD) compared to Group 3 at 8 weeks.

New bone formation in rabbits maxillary sinus after augmentation with demineralized particulated human tooth graft (DHT) was evaluated by comparing its histomorphometric outcomes to sinus augmented with Bio-Oss ${ }^{\circledR}$ (deproteinized bovine bone) in a study conducted by $\mathrm{Xu}$ et al. in 2018 [21]. They performed bilateral sinus augmentation in 8 adult male rabbits which were divided to control group and experimental group. The ratio of new bone formation in the Bio-Oss ${ }^{\circledR}$ group at 2 and 8 weeks was 5.94 $(0.36) \%$ and $4.83(0.41) \%$, respectively, while in the DHT group, significantly higher ratio was observed with $5.54(0.5) \%$ and $19.45(2.06) \%$, at 2 and 8 weeks respectively. There were significantly lesser amounts of tooth graft material at 8 weeks compared to 2 weeks while in the control group the decrease in graft material amount over time was lesser. 
Table 6. Histomorphometric analysis: humans

\begin{tabular}{|c|c|c|c|c|}
\hline Study & $\begin{array}{c}\text { New bone formation } \\
(\%)\end{array}$ & $\begin{array}{c}\text { Residual graft material } \\
(\%)\end{array}$ & $\begin{array}{c}\text { Marrow space } \\
(\%)\end{array}$ & $\begin{array}{l}\text { Osteoid thickness } \\
(\mu \mathrm{m})\end{array}$ \\
\hline \multirow{3}{*}{ Jun et al. [15] } & $\begin{array}{c}\text { AutoBT: } \\
31.07(14.52) \\
\end{array}$ & $\begin{array}{c}\text { AutoBT: } \\
29(10.27)\end{array}$ & $\begin{array}{c}\text { AutoBT: } \\
39.93(18.92) \\
\end{array}$ & $\begin{array}{c}\text { AutoBT: } \\
13.12(5.16)\end{array}$ \\
\hline & $\begin{array}{c}\text { Bio-Oss }{ }^{\circledR}: \\
26.49(7.13) \\
\end{array}$ & $\begin{array}{c}\text { Bio-Oss }{ }^{\circledR}: \\
31.12(14.51) \\
\end{array}$ & $\begin{array}{c}\text { Bio-Oss }{ }^{\circledR:}: \\
42.38(16.37) \\
\end{array}$ & $\begin{array}{l}\text { Bio-Oss }^{\circledR}: \\
8.35(3.94) \\
\end{array}$ \\
\hline & $\begin{array}{l}\text { No difference } \\
(\mathrm{P}=0.556)\end{array}$ & $\begin{array}{l}\text { No difference } \\
(\mathrm{P}=0.896)\end{array}$ & $\begin{array}{l}\text { No difference } \\
(\mathrm{P}=0.471)\end{array}$ & $\begin{array}{l}\text { Significant difference } \\
\quad(\mathrm{P}=0.025)\end{array}$ \\
\hline \multirow{3}{*}{ Kim et al. [18] } & $\begin{aligned} & \text { AutoFDT block } \\
&+ \text { PRP: } 23.13(1.42) \\
&\end{aligned}$ & $\begin{aligned} & \text { AutoFDT block } \\
&+ \text { PRP: } 22.21(1.19) \\
&\end{aligned}$ & \multirow{3}{*}{ Not evaluated } & \multirow{3}{*}{ Not evaluated } \\
\hline & $\begin{array}{c}\text { Allograft and xenograft } \\
\text { powder with PRP: } \\
24.18(2.19)\end{array}$ & $\begin{array}{l}\text { Allograft and xenograft } \\
\text { powder with PRP: } \\
31.18(2.09)\end{array}$ & & \\
\hline & $\begin{array}{c}\text { No difference } \\
(\mathrm{P}=0.548)\end{array}$ & $\begin{array}{l}\text { Significant difference } \\
\quad(\mathrm{P}=10.008)^{\mathrm{a}}\end{array}$ & & \\
\hline
\end{tabular}

aSignificant difference between block and powder was observed - larger spaces between the blocks compared to powder. AutoFDT = autogenous fresh demineralized tooth; autoBT = autogenous tooth bone graft; PRP = platelet rich plasma.

Table 7. Histomorphometric analysis: animal

\begin{tabular}{|c|c|c|c|}
\hline Study & $\begin{array}{c}\text { New bone formation } \\
(\%)\end{array}$ & $\begin{array}{c}\text { Residual graft material } \\
(\%)\end{array}$ & $\begin{array}{c}\text { Lamellar bone } \\
(\%) \\
\end{array}$ \\
\hline \multirow{3}{*}{ Lee et al. [19] } & Autogenous teeth: $57.19(11.16)$ & \multirow{3}{*}{ Not evaluated } & \multirow{3}{*}{ Not evaluated } \\
\hline & $\begin{array}{c}\text { Synthetic hydroxyapatite: } 34.07 \\
\text { (13.09) }\end{array}$ & & \\
\hline & No difference $(\mathrm{P}>0.05)^{\mathrm{a}}$ & & \\
\hline \multirow{5}{*}{ Sohn et al. [20] } & $\begin{array}{c}\text { Blood clots: } \\
2 \text { weeks: } 8.46(1.88) \text {, } \\
4 \text { weeks: } 17.8(2.63), \\
8 \text { weeks: } 12.1(2.71)\end{array}$ & $\begin{array}{l}\text { Blood clots: } \\
\text { 2, 4, } 8 \text { weeks: not } \\
\text { evaluated }\end{array}$ & $\begin{array}{l}4 \text { weeks: } 2.21(0.69) \text {, } \\
8 \text { weeks: } 10.45(2.1)\end{array}$ \\
\hline & $\begin{array}{l}\text { Anorganic bovine bone: } \\
2 \text { weeks: } 16.09(1.52) \text {, } \\
4 \text { weeks: } 18.91(1.96), \\
8 \text { weeks: } 19.65(1.81)\end{array}$ & $\begin{array}{l}\text { Anorganic bovine bone: } \\
\text { 2 weeks: } 36.74 \text { (3.94), } \\
\text { 4 weeks: } 32.97 \text { (2.59), } \\
8 \text { weeks: } 35.21(3.16)\end{array}$ & $\begin{array}{l}4 \text { weeks: } 1.48(0.3) \text {, } \\
8 \text { weeks: } 4.85(0.47)\end{array}$ \\
\hline & $\begin{array}{c}\beta \text {-ТСР: } \\
\text { 2 weeks: } 15.05(1.21) \text {, } \\
\text { 4 weeks: } 20.13(2.3), \\
8 \text { weeks: } 20.73(1.99)\end{array}$ & $\begin{array}{c}\beta \text {-ТСР: } \\
\text { 2 weeks: } 37.19(3.08) \text {, } \\
\text { 4 weeks: } 29.43(3.2), \\
8 \text { weeks: } 21.01(3.4)\end{array}$ & $\begin{array}{l}4 \text { weeks: } 1.46(0.4) \text {, } \\
8 \text { weeks: } 4.04(0.52)\end{array}$ \\
\hline & $\begin{array}{c}\text { DTD: } \\
\text { 2 weeks: } 17.71(2.2), \\
\text { 4 weeks: } 20.73(1.99) \text {, } \\
\text { 8 weeks: } 28.09(1.51)\end{array}$ & $\begin{array}{c}\text { DTD: } \\
\text { 2 weeks: } 44.41(5.26) \text {, } \\
\text { 4 weeks: } 28.25(3.68) \text {, } \\
8 \text { weeks: } 14.04(3.17)\end{array}$ & $\begin{array}{l}4 \text { weeks: } 1.31(0.3) \text {, } \\
8 \text { weeks: } 4.7(0.59)\end{array}$ \\
\hline & $\begin{array}{l}\text { At } 8 \text { weeks: } \\
\text { significant greater in DTD } \\
\text { group than } \beta \text {-TCP group }\end{array}$ & $\begin{array}{l}\text { DTD: significantly less } \\
\text { than Bio-Oss }{ }^{\circledR} \\
\text { or } \beta \text {-TCP area at } 8 \text { weeks } \\
(\mathrm{P}<0.05)\end{array}$ & $\begin{array}{l}\text { Significantly higher } \\
\text { in blood clots group } \\
(\mathrm{P}<0.5)\end{array}$ \\
\hline \multirow{3}{*}{ Xu et al. [21] } & $\begin{array}{c}\text { DHT: } \\
\text { 2 weeks: } 5.54(0.5), \\
8 \text { weeks: } 19.45(2.06)\end{array}$ & $\begin{array}{c}\text { DHT: } 41.01(2.23) \text { and } \\
9.62(1.02) ; \mathrm{P}<0.5\end{array}$ & 8 weeks: $6.08(0.65)$ \\
\hline & $\begin{array}{c}\text { Bio-Oss }{ }^{\circledR}: \\
\text { 2 weeks: } 5.94(0.36) \text {; } \\
8 \text { weeks: } 4.83(0.41)\end{array}$ & $\begin{array}{c}\text { Bio-Oss }^{\mathbb{B}}: \\
2 \text { weeks: } 29.03 \text { (2.32), } \\
8 \text { weeks: } 25.53(2.15)\end{array}$ & 8 weeks: $5.36(0.45)$ \\
\hline & $\begin{array}{l}\text { Significantly higher in DHT } \\
\text { group }\end{array}$ & - & $\begin{array}{l}\text { Increased amount in } \\
\text { DHT }(\mathrm{P}<0.5)\end{array}$ \\
\hline
\end{tabular}

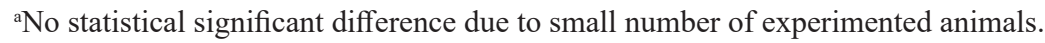

$\mathrm{DHT}=$ demineralized human particulate tooth bone; $\mathrm{DTD}=$ demineralized tooth dentin; $\beta$-TCP $=\beta$-tricalcium phosphate. 
The researchers also reported on an increased amount and density of mature lamellar bone with higher connections of tooth graft and the new bone in tooth graft group compared to Bio-Oss ${ }^{\circledR}$.

\section{Histologic findings}

The following histological findings were seen in groups who received maxillary sinus bone grafting with material derived from teeth.

\section{Human studies}

Mature lamellar bone integration of residual bone with maxillary sinus was seen in the study of Jun et al. [15]. Enamel and dentin parts of graft were surrounded by formation of bone, as well as new bone bridges between graft materials. AutoBT sample also showed new bone formed covered with osteoblasts and mature lamellar bone surrounding the graft. Active woven bone and medullary space formation was detected also.

The study of Kim et al. [18] demonstrated new bone formation which was filling the empty spaces between the autoFDT blocks. Regular presence of osteocytes with equal distribution in the new bone area was detected, as well as direct integration between the new bone and autoFDT.

\section{Animal studies}

The study of Lee et al. [19] reported growth of new bone at the sides of tooth derived graft material, as well as osteoclastic activity of multinucleated giant cells indicating bone remodelling.

Growth of new bone was seen along maxillary sinus cavity in the study of Sohn et al. [20], surrounded with many osteoblasts. At 4 weeks - there was increase in bone growth on DTD surface and in central area of the space. The formed bone was thicker than the bone at 2 weeks, more vascularized and with more connective tissue. DTD reduced in its density compared with 2 weeks. At 8 weeks, highly increased thickness and density of new bone was identified. Many osteoblasts were seen around the new bone and some osteoclasts around the DTD, which decreased in size compared to 4 weeks.

$\mathrm{Xu}$ et al. [21] found new bone growth at 2 weeks on the DHT surface, with osteoblasts on its surface. At 8 weeks - significant increase in density and thickness of new bone was seen. Presence of large amount of mature lamellar bone inside the new formed bone, marrow space containing adipose tissue. Decrease in size and density of DHT compared to 2 weeks.
In comparison with control group, the experimental group had active bone resorption and formation, with presence of osteoclasts inside and on the surface of DHT.

\section{Complications}

Kim et al. [17] reported no serious complications or implant failures following autoBT sinus grafting, similar results were observed by Kim et al. [18] reporting no sinusitis, implant loss or other complications. There was no bone graft exposure, infection or edema reported by Lee et al. [19], and signs of postoperative inflammation in the animal study of Sohn et al. [20] .

\section{DISCUSSION}

Following augmentation of the maxillary sinus, the new formed sinus floor is being continuously resorbed by the repneumatization phenomenon $[\underline{22}, \underline{23}]$, which in turn can lead to failure of the implant through exposure of its apical portion to sinus mucosa, instead of the implant being in contact with bone. Thus, maintaining stability of an implant placed in the maxillary sinus area requires a bone graft material which shows minimal resorption rates for a satisfactory long period of time [24,25]. However, when a graft material like anorganic bovine bone, which is very slow resorbable [26], has a low remodelling capacity, it occupies space and prevents new bone formation $[\underline{27}, \underline{28}]$.

In an attempt to predict the success or failure of a graft material, learning its decrease in height is more decisive than measuring decrease in its volume [29], and since precise measurement of height decrease is possible with panoramic radiography [30], we reviewed studies which investigated height decrease of tooth graft materials and compared their resulting parameters with xenografts, allografts and alloplasts using panoramic radiography. The height decrease parameter of tooth bone graft was not significantly different from that of DFDBA, DBBM, Osteon ${ }^{\mathrm{TM}}$ and combined allograft and xenograft powder with PRP in 3 different studies [16-18], suggesting that it might have similar prognosis as the materials compared in each study.

Based on 2 human studies in our review the bone formation capacity of autoBT was similar to that of Bio-Oss ${ }^{\circledR}[15]$ and to that of combination of allograft and xenograft [18] when used in maxillary sinus. In addition, osteoid thickness in autoBT group was significantly higher than Bio-Oss ${ }^{\circledR}$ group [15], 
a finding which might imply that autoBT is not inferior to Bio-Oss ${ }^{\circledR}$ in its bone formation capacity. Improved capacity of new bone generation was reported in 2 animal studies among tooth bone graft groups when compared to Bio-Oss ${ }^{\circledR}$ and $\beta$-TCP $[19,21]$, while no difference was found in a porcine study of Lee et al. [19] when compared to synthetic hydroxyapatite.

High crystalline content graft materials show poor osteoconductive properties, because they are harder for osteoclasts to degrade. AutoBT made of crown is a high crystalline material unlike root autoBT, which has a low-crystalline calcium phosphate compositionknown to have osteoinductive and osteoconductive properties $[\underline{31}, \underline{32}]$.

Regarding the implant success, Kim et al. [18] reported $100 \%$ implant success in the autoFDT block group after 2.5 years. Nevertheless, the reviewed studies failed to provide long enough follow-up period (the longest was 2.5 years [18]), to conclude any valuable prediction. Kim et al. [33] reported no abnormal reaction in hypersensitivity and cytotoxicity test after using particulated dentin. Complications such as infections and inflammatory reactions of sinus mucosa were not encountered in any of the studies reviewed in the present paper.

The maxillary sinus presents an environment which forms good bony housing, as it has bone and mucous membrane in close proximity (in the upper area). Furthermore, some studies have proved that limited bony formation takes place even in a graftless sinus augmentation $[34,35]$. Therefore, in the process of comparing different graft materials which are used for sinus elevation, the differences between the materials are blurred [16], and this might be the reason for the similar or resembling results which were observed among the reviewed studies- this is one limitation of our review. Another important drawback is the relatively small number of subjects which were tested in each study; a drawback which gains even more influence considering the first limitation discussed previously.

\section{CONCLUSIONS}

Tooth derived bone graft materials which were used in the maxillary sinus showed similar resorption heights with allografts, xenografts and alloplasts in the reviewed human studies, in addition to similar or even better bone formation capacity compared to those graft materials in the reviewed animal studies. Within the previously presented limitations of our review, we suggest that tooth derived graft materials are as successful as xenografts, allografts and alloplasts in sinus augmentation procedures according to the radiographic and histomorphometric showings. Additional wider research should be conducted in order to determine whether tooth derived graft materials are superior to the currently used materials.

\section{ACKNOWLEDGMENTS AND DISCLOSURE STATEMENTS}

The authors report no conflicts of interest related to the present review.

\section{REFERENCES}

1. Dental implants facts and figures. American Academy of Implant Dentistry. [URL: https://www.aaid.com/about/press room/dental implants faq.html]

2. Tatum H Jr. Maxillary and sinus implant reconstructions. Dent Clin North Am. 1986 Apr;30(2):207-29. [Medline: $\underline{3516738]}$

3. Mohan N, Wolf J, Dym H. Maxillary sinus augmentation. Dent Clin North Am. 2015 Apr;59(2):375-88. [Medline: 25835800] [doi: $10.1016 /$ j.cden.2014.10.001]

4. Ting M, Rice JG, Braid SM, Lee CYS, Suzuki JB. Maxillary Sinus Augmentation for Dental Implant Rehabilitation of the Edentulous Ridge: A Comprehensive Overview of Systematic Reviews. Implant Dent. 2017 Jun;26(3):438-464. [Medline: 28520572] [doi: 10.1097/ID.0000000000000606]

5. Albrektsson T, Johansson C. Osteoinduction, osteoconduction and osseointegration. Eur Spine J. 2001 Oct;10 Suppl 2:S96-101. [Medline: 11716023] [PMC free article: 3611551] [doi: 10.1007/s005860100282]

6. Nampo T, Watahiki J, Enomoto A, Taguchi T, Ono M, Nakano H, Yamamoto G, Irie T, Tachikawa T, Maki K. A new method for alveolar bone repair using extracted teeth for the graft material. J Periodontol. 2010 Sep;81(9):1264-72. [Medline: 20476887] [doi: 10.1902/jop. 2010.100016]

7. Park M, Mah YJ, Kim DH, Kim ES, Park EJ. Demineralized deciduous tooth as a source of bone graft material: its biological and physicochemical characteristics. Oral Surg Oral Med Oral Pathol Oral Radiol. 2015 Sep;120(3):307-14. [Medline: 26297390] [doi: 10.1016/j.0000.2015.05.021] 
8. Koga T, Minamizato T, Kawai Y, Miura K, I T, Nakatani Y, Sumita Y, Asahina I. Bone Regeneration Using Dentin Matrix Depends on the Degree of Demineralization and Particle Size. PLoS One. 2016 Jan 21;11(1):e0147235. [Medline: 26795024] [PMC free article: 4721666] [doi: 10.1371/journal.pone.0147235]

9. Kühl S, Götz H, Brochhausen C, Jakse N, Filippi A, d'Hoedt B, Kreisler M. The influence of substitute materials on bone density after maxillary sinus augmentation: a microcomputed tomography study. Int J Oral Maxillofac Implants. 2012 Nov-Dec;27(6):1541-6. [Medline: 23189308]

10. Kim YK, Lee J, Um IW, Kim KW, Murata M, Akazawa T, Mitsugi M. Tooth-derived bone graft material. J Korean Assoc Oral Maxillofac Surg. 2013 Jun;39(3):103-11. [Medline: 24471027] [PMC free article: 3858164] [doi: $10.5125 /$ jkaoms.2013.39.3.103]

11. Moher D, Liberati A, Tetzlaff J, Altman DG. PRISMA Group. Preferred reporting items for systematic reviews and meta-analyses: the PRISMA statement. Int J Surg. 2010;8(5):336-41. Erratum in: Int J Surg. 2010;8(8):658. [doi: 10.1016/j.ijsu.2010.07.299]

12. Higgins JPT, Green S. Cochrane Handbook for Systematic Reviews of Interventions version 5.1.0. The Cochrane Collaboration, 2011. [URL: http://training.cochrane.org/handbook]

13. Jeong KI, Kim SG, Kim YK, Oh JS, Jeong MA, Park JJ. Clinical study of graft materials using autogenous teeth in maxillary sinus augmentation. Implant Dent. 2011 Dec;20(6):471-5. [Medline: 22067601] [doi: 10.1097/ID.0b013e3182386d74]

14. Pohl V, Schuh C, Fischer MB, Haas R. A New Method Using Autogenous Impacted Third Molars for Sinus Augmentation to Enhance Implant Treatment: Case Series with Preliminary Results of an Open, Prospective Longitudinal Study. Int J Oral Maxillofac Implants. 2016 May-Jun;31(3):622-30. [Medline: 27183071] [doi: 10.11607/jomi.4172]

15. Jun SH, Ahn JS, Lee JI, Ahn KJ, Yun PY, Kim YK. A prospective study on the effectiveness of newly developed autogenous tooth bone graft material for sinus bone graft procedure. J Adv Prosthodont. 2014 Dec;6(6):528-38. [Medline: 25551014] [PMC free article: $\underline{4279053}$ ] [doi: 10.4047/jap.2014.6.6.528]

16. Jeong TM, Lee JK. The Efficacy of the Graft Materials after Sinus Elevation: Retrospective Comparative Study Using Panoramic Radiography. Maxillofac Plast Reconstr Surg. 2014 Jul;36(4):146-53. [Medline: 27489826] [PMC free article: 4281913] [doi: 10.14402/jkamprs.2014.36.4.146]

17. Kim YK, Lee J, Yun JY, Yun PY, Um IW. Comparison of autogenous tooth bone graft and synthetic bone graft materials used for bone resorption around implants after crestal approach sinus lifting: a retrospective study. J Periodontal Implant Sci. 2014 Oct;44(5):216-21. [Medline: 25368809] [PMC free article: 4216397] [doi: 10.5051/jpis.2014.44.5.216]

18. Kim ES, Kang JY, Kim JJ, Kim KW, Lee EY. Space maintenance in autogenous fresh demineralized tooth blocks with platelet-rich plasma for maxillary sinus bone formation: a prospective study. Springerplus. 2016 Mar 7;5:274. [Medline: 27047706] [PMC free article: 4779789] [doi: 10.1186/s40064-016-1886-1]

19. Lee du H, Yang KY, Lee JK. Porcine study on the efficacy of autogenous tooth bone in the maxillary sinus. J Korean Assoc Oral Maxillofac Surg. 2013 Jun;39(3):120-6. [Medline: 24471029] [PMC free article: 3858170] [doi: $10.5125 / \mathrm{jkaoms} .2013 .39 .3 .120$ ]

20. Sohn DS, Moon YS. Histomorphometric study of rabbit's maxillary sinus augmentation with various graft materials. Anat Cell Biol. 2018 Dec;51(Suppl 1):S1-S12. [Medline: 30680247] [PMC free article: 6342707] [doi: 10.5115/acb.2018.51.S1.S1]

21. Xu X, Sohn DS, Kim HG, Lee SJ, Moon YS. Comparative Histomorphometric Analysis of Maxillary Sinus Augmentation With Deproteinized Bovine Bone and Demineralized Particulate Human Tooth Graft: An Experimental Study in Rabbits. Implant Dent. 2018 Jun;27(3):324-331. [Medline: 29613862] [doi: 10.1097/ID.0000000000000755]

22. Jensen OT, Shulman LB, Block MS, Iacono VJ. Report of the Sinus Consensus Conference of 1996. Int J Oral Maxillofac Implants. 1998;13 Suppl:11-45. [Medline: 9715571]

23. Nyström E, Legrell PE, Forssell A, Kahnberg KE. Combined use of bone grafts and implants in the severely resorbed maxilla. Postoperative evaluation by computed tomography. Int J Oral Maxillofac Surg. 1995 Feb;24(1 Pt 1):20-5. [Medline: 7782636] [doi: 10.1016/S0901-5027(05)80851-3]

24. GaRey DJ, Whittaker JM, James RA, Lozada JL. The histologic evaluation of the implant interface with heterograft and allograft materials--an eight-month autopsy report, Part II. J Oral Implantol. 1991;17(4):404-8. [Medline: 1813648]

25. Romero-Millán J, Hernández-Alfaro F, Peñarrocha-Diago M, Soto-Peñaloza D, Peñarrocha-Oltra D, PeñarrochaDiago MA. Simultaneous and delayed direct sinus lift versus conventional implants: Retrospective study with 5-years minimum follow-up. Med Oral Patol Oral Cir Bucal. 2018 Nov 1;23(6):e752-e760. [Medline: 30341266] [PMC free article: 6261006] [doi: 10.4317/medoral.22612]

26. Traini T, Valentini P, Iezzi G, Piattelli A. A histologic and histomorphometric evaluation of anorganic bovine bone retrieved 9 years after a sinus augmentation procedure. J Periodontol. 2007 May;78(5):955-61. [Medline: 17470032] [doi: 10.1902/jop.2007.060308]

27. Sohn DS, Kim WS, An KM, Song KJ, Lee JM, Mun YS. Comparative histomorphometric analysis of maxillary sinus augmentation with and without bone grafting in rabbit. Implant Dent. 2010 Jun;19(3):259-70. [Medline: 20523182] [doi: 10.1097/ID.0b013e3181df1406]

28. Sohn DS, Moon JW, Lee WH, Kim SS, Kim CW, Kim KT, Moon YS. Comparison of new bone formation in the maxillary sinus with and without bone grafts: immunochemical rabbit study. Int J Oral Maxillofac Implants. 2011 Sep-Oct;26(5):1033-42. [Medline: 22010087] 
29. Hatano N, Shimizu Y, Ooya K. A clinical long-term radiographic evaluation of graft height changes after maxillary sinus floor augmentation with a 2:1 autogenous bone/xenograft mixture and simultaneous placement of dental implants. Clin Oral Implants Res. 2004 Jun;15(3):339-45. [Medline: 15142097] [doi: 10.1111/j.1600-0501.2004.00996.x]

30. Hallman M, Hedin M, Sennerby L, Lundgren S. A prospective 1-year clinical and radiographic study of implants placed after maxillary sinus floor augmentation with bovine hydroxyapatite and autogenous bone. J Oral Maxillofac Surg. 2002 Mar;60(3):277-84; discussion 285-6. [Medline: 11887139] [doi: 10.1053/joms.2002.30576]

31. Kim GW, Yeo IS, Kim SG, Um IW, Kim YK. Analysis of crystalline structure of autogenous tooth bone graft material: X-Ray diffraction analysis. J Korean Assoc Oral Maxillofac Surg. 2011 Jun;37(3):225-8. [doi: 10.5125/jkaoms.2011.37.3.225]

32. Kim YK, Lee HJ, Kim KW, Kim SG, Um IW. Guide bone regeneration using autogenous teeth: case reports. J Korean Assoc Oral Maxillofac Surg. 2011 Apr;37(2):142-7. [doi: 10.5125/jkaoms.2011.37.2.142]

33. Kim YK, Kim SG, Lee JH. Cytotoxicity and hypersensitivity test of toothash. J Korean Assoc Maxillofac Plast Reconstr Surg. 2001 Sep;23(5):391-5.

34. In YS, Park YW. Factors affecting survival of maxillary sinus augmented implants. J Korean Assoc Maxillofac Plast Reconstr Surg. 2011 May;33(3):241-8.

35. Del Fabbro M, Testori T, Francetti L, Weinstein R. Systematic review of survival rates for implants placed in the grafted maxillary sinus. J Pros Dent. 2005 Sep;94(3): 266. [doi: 10.1016/j.prosdent.2005.04.024]

\section{To cite this article:}

Shavit E, Shavit I, Pinchasov D, Shavit D, Pinchasov G, Juodzbalys G.

The Use of Tooth Derived Bone Graft Materials in Sinus Augmentation Procedures: a Systematic Review

J Oral Maxillofac Res 2019;10(2):e1

URL: http://www.ejomr.org/JOMR/archives/2019/2/e1/v10n2e1.pdf

doi: $10.5037 /$ jomr.2019.10201

Copyright (C) Shavit E, Shavit I, Pinchasov D, Shavit D, Pinchasov G, Juodzbalys G. Published in the JOURNAL OF ORAL \& MAXILLOFACIAL RESEARCH (http://www.ejomr.org), 30 June 2019.

This is an open-access article, first published in the JOURNAL OF ORAL \& MAXILLOFACIAL RESEARCH, distributed under the terms of the Creative Commons Attribution-Noncommercial-No Derivative Works 3.0 Unported License, which permits unrestricted non-commercial use, distribution, and reproduction in any medium, provided the original work and is properly cited. The copyright, license information and link to the original publication on (http://www.ejomr.org) must be included. 